\title{
Studies of microscopic strains on Alloy 600 surfaces arising from stress corrosion cracking
}

\author{
N. S. McIntyre ${ }^{1}$, T. Simpson ${ }^{1}$ J. Qin ${ }^{1}$, N. Sherry ${ }^{1}$, M. Bauer ${ }^{1}$, \\ J. Ulaganathan ${ }^{2}$, A. G. Carcea ${ }^{2}$, R. C. Newman ${ }^{2}$, M. Kunz ${ }^{3}$ \\ \& N. Tamura $^{3}$ \\ ${ }^{1}$ Faculty of Science, University of Western Ontario, Canada \\ ${ }^{2}$ University of Toronto, Canada \\ ${ }^{3}$ Advanced Light Source, Lawrence Berkeley National Laboratory, USA
}

\begin{abstract}
Microscopic distributions of elastic and plastic strains have been studied in Alloy 600 during accelerated corrosion. Laue diffraction is employed with a submicron beam of highly coherent polychromatic (white) radiation. The diffraction patterns are analysed to detect elastic and plastic deformations associated with the crack initiation and propagation processes. Stressed C ring and unstressed mill annealed samples of Alloy 600 were corroded under hydrothermal controlled electrochemical conditions. In the $\mathrm{C}$ ring, the filamentous surface cracks produced had compressive strain fields along each crack. In the mill annealed sample, changes in strain fields in the same area were measured as oxidation progressed. Cracking at the metal grain boundaries appeared to be induced by expansive growth of surface oxides. For the mill annealed sample, accumulation of elastic strains in the grain boundaries appeared in advance of crack propagation.
\end{abstract}

Keywords: strain, stress corrosion cracking, Laue diffraction.

\section{Introduction}

Stress corrosion cracking (SCC) is the single most common mode of degradation for high performance nickel alloys. SCC of nickel and its alloys is generally believed to begin at an interface, possibly resulting from stresses arising from the expansion of corrosion products in a grain boundary that can interact with local strains already present in the material. 
These and other stress-related properties are being studied using microscopic beams of highly coherent radiation that interact with the material to produce Laue diffraction patterns for each micron-size area. Because of the low scattering properties of such beams it is possible to derive much more accurate information about crystal properties, such as deviations from the "classic" properties of the unit cell that exist only in perfect crystals. From deviatoric changes to the lengths of the unit cell, one can determine the spatial distribution (residual) elastic strain tensors in a material; from the deviatoric changes to angles in the unit cell, information about changes to the plastic deformation can be derived including the local density and direction of dislocations [1-5]. Each Laue x-ray pattern can now be more accurately assessed than is possible with related electron beam techniques like Electron Backscattering Diffraction (EBSD).

Our initial research in this area was to study local strains in Alloy 600 (16\%Cr $8 \%$ Fe balance Ni) that had been deformed plastically [6, 7]. Since then, a reproducible electrochemical protocol has been refined that has caused Alloy 600 tubing $\mathrm{C}$ rings to undergo accelerated SCC in an aqueous caustic solution. Alloy 600 tubing has been widely used to transport high temperature coolant in nuclear power steam generators. Such tubing has frequently been found to undergo stress corrosion cracking in the steam generators of nuclear power stations particularly in areas where the where the tubing is supported mechanically $[8,9]$.

This present study has concentrated on the detection of the initial steps in the process of SCC: the accumulation of stresses at the surface of the alloy sample and the first formation of surface and internal oxides that could initiate a crack. Using experiences from previous experiments, corrosion conditions have been chosen that would allow a measured stepwise approach to crack initiation that would show incremental changes to the strain and dislocation distributions. Two different sample geometries have been used: a pre-stressed C ring [6] and a mill annealed polished flat with little stress. In both samples cracking has been induced that could be considered to be at the incipient stage. Laue studies of both have explored the local elastic strain fields near the crack and, in the case of the flat, strain changes in the both the surface oxide and the metal substrate have been followed during three steps in the corrosion process.

\section{Experimental}

The C-ring was prepared from Alloy 600 nuclear grade tubing according to ASTM standard G-38. The stress applied was equivalent to a calculated strain of $2 \%$. The flat was prepared from a mill annealed ingot of Alloy 600 . The surface was mechanically polished to a 0.5 micron diamond grit finish. Then an area of several hundred square microns was cleaned and polished with a focused gallium ion beam (FIB). The corrosion test of the C-ring was conducted so that, based on past practice $[10,11]$ any stress corrosion cracking of the sample would be slight. The $\mathrm{C}$ ring was exposed in its stressed condition in an autoclave containing a solution of $10 \mathrm{wt} . \% \mathrm{NaOH}$ in deionized water at a temperature of 315 C. All tests were conducted in a $0.5 \mathrm{l}$ nickel beaker (liner) containing $230 \mathrm{ml}$ 
of solution inside an alloy-625 autoclave. Prior to heating the autoclave, the caustic solution was de-aerated by nitrogen for at least $12 \mathrm{~h}$. After temperature stabilization, a potential of $150 \mathrm{mV}$ vs. pseudo-RE (Alloy 600) was applied to the whole sample (C-ring, bolt and washer assembly) and the total current density was monitored. After initial decay from $\sim 150 \mu \mathrm{A} \mathrm{cm}{ }^{-2}$ the current densities finally reached values of $\sim 30 \mu \mathrm{A} \mathrm{cm} \mathrm{cm}^{-2}$ after an exposure time of 120 minutes. For corrosion of the flat, a potential of $100 \mathrm{mV}$ was used; three separate exposures were carried out for periods of 12, 12 and 24h with a microscopic Laue diffraction study of the same area after each exposure. Following the corrosion test the sample microstructure was studied by SEM and was sectioned by a Focused Ion Beam to obtain a better view of the grain structure and crack propagation.

Laue studies were done at beamline 12.3.2 at the Advanced Light Source synchrotron using conditions reported in a previous paper [7]. The polychromatic beam size was 1 micron diameter. The Laue images were processed using FOXMAS software (Fast On-line X-ray Microdiffraction Analysis Software) [12]. FOXMAS is parallelized software that is partially based on the XMAS [2] source code and is capable of processing the same images nearly two orders of magnitude faster than XMAS. The software has been coded to run on IBM InfoSphere Streams computing software (version 2.0) on processors at the University of Western Ontario. Indexing was done using a centroid fit of the detected spots and was also re-indexed to show all results with 7 or more spots indexed. The spots were indexed for fcc nickel with a cell parameter of $0.35564 \mathrm{~nm}$. in Alloy 600 [13]. Angular mis-orientation information about plastic deformation was also collected using Laue diffraction and these data were computed and displayed using software developed by this group called "Skew".

\section{Results}

\subsection{C ring stress corrosion cracking}

The apex of the outside tube diameter proved to have a significant fraction of grain boundaries that appeared to be beveled due to enhanced oxidation. The sub-surface structure of such boundaries was explored using a FIB to cut a cross section (see Figure 1). A lateral $3 \mathrm{kV}$ SEM image of a FIB cut is shown in Figure 1(a), revealing the oxide metal interface, the beveled grain boundary/surface interfaces and detail of the surface oxide. A crack pathway can be seen in the oxide surface, as well as through the compact oxide in the bevel and along the boundary.

It appears that the enhanced oxidation resulted in crack propagation that extended through the compact oxide in the bevels, the oxide crystals on the outer surface and into the grain boundaries below the bevels. This appears to be good circumstantial evidence for the initiation of cracking from the expansive growth of oxides within the bevels. 
(a)

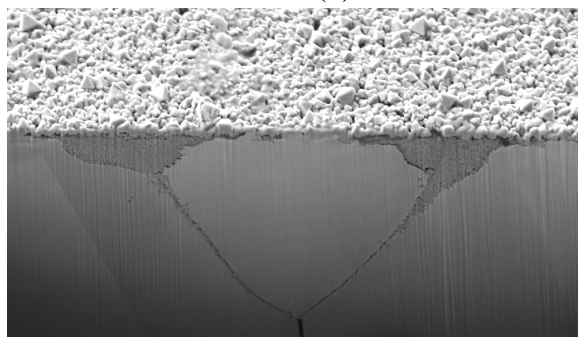

(b)

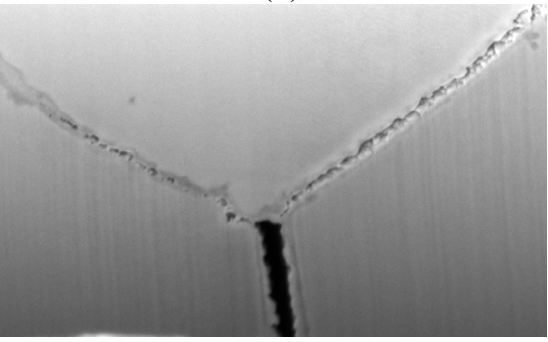

Figure 1: SEM images of grain boundary structure along the apex of the outside diameter of the $\mathrm{C}$ ring (a) lateral view of a FIB cut revealing the oxide metal interface. (b) detail of the triple point shown in the lower part of (a).

The strains associated with this cracking process were studied with the aid of a Laue diffraction map that was done on a narrow (21 micron) strip along the top of the apex in the vicinity of a number of the cracks. A limited width was required because of the stigmatic limitations of the $\mathrm{X}$ ray optics. No exact match was possible between the coordinates of an individual crack and mapped result.

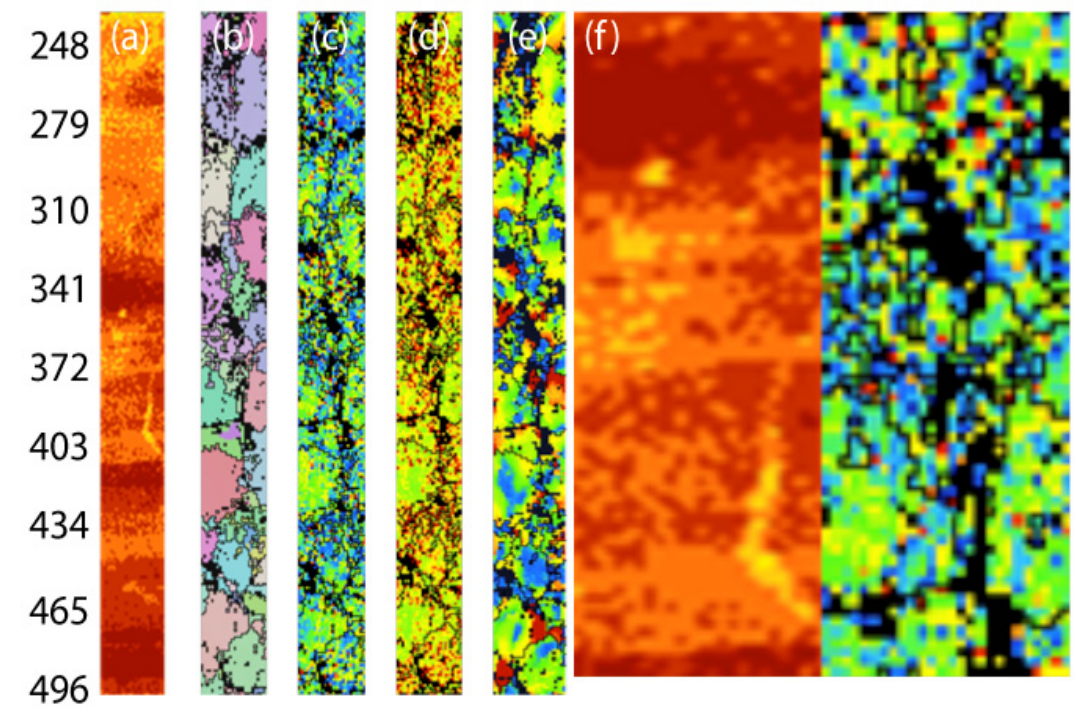

Figure 2: $\quad$ Laue diffraction maps of a narrow portion of the apex of the outside diameter of the $\mathrm{C}$ ring.(a) absorbed $\mathrm{x}$ ray image (b) crystallographic orientation image with grain boundaries outlined by black lines (c) y elastic strain tensor image (d) composite elastic strain image (e) absolute mis-orientation image (f) expanded view of absorbed $\mathrm{x}$ ray map and y strain tensor map. 
However, an overlap between a physical crack and the PXM map was made possible from the use of imaging $\mathrm{x}$ ray absorption measurements as outlined in a previous publication [7]. Figure 2 shows maps of the imaging $\mathrm{x}$ ray image along with those of orientation, Y stain tensor, composite strain and absolute misorientation. In Figure 2(a) the light streaks are ascribed to near surface cracks.

By comparing the locus of this discontinuity with the orientation map (Figure 2(b)), it appears that the crack is aligned with the boundary between two grains. A line of boundaries continue up the centre of the map and along this line a number of pixels cannot be indexed, suggesting significant disruption of the crystal structure along this line, some of which must be due to plastic strain caused by the cracking. The map of y tensor strain (Figure 2(c)) shows an accumulation of compressive elastic strain in the narrow band in grains to the left of the boundary and lying close to it. Figure 2(e) shows the distributions of misorientation near the crack region. Compared to the y tensor elastic strain map there is little evidence of correlation between the crack locus and incidence of plastic strain as measured by absolute mis-orientation.

A magnification in Figure 2(f) of the presumed crack locus and the strain pattern shows that most of the compressive elastic strain is found within several microns to one side of the crack. This pattern is also observed in the composite strain map (Figure 2(d)) but not as clearly-defined as in the y tensor map. The compressive elastic strain intensity reaches a maximum of $\sim 5 \times 10-3$ within a few microns of the crack itself. The immediate region of the crack has a low crystal order as diffraction patterns there cannot be indexed and appear as black in the Figure.

\subsection{Mill annealed polished flat}

A small region on the polished surface of the flat was pre-treated with a focussed ion beam. This was done to allow minute changes in the physical structure of the flat to be followed during the three corrosion treatments. This proved to be very useful in identifying the onset of cracking after the third

(a)

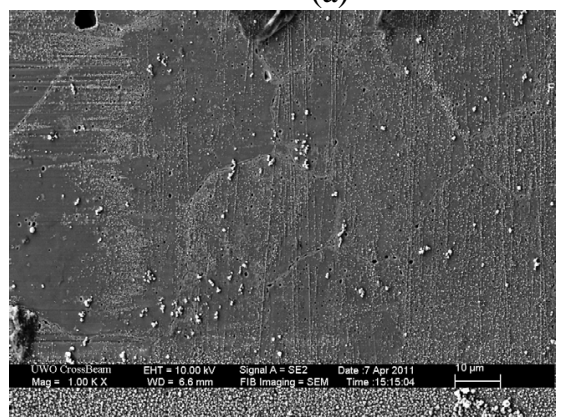

(b)

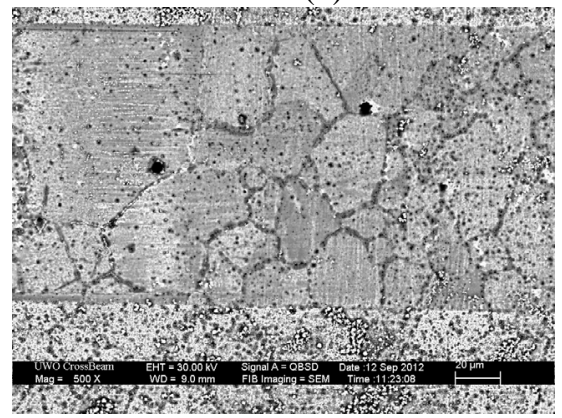

Figure 3: $\quad$ SEM plan view images of the surface of the corrosion flat after the (a) second treatment (secondary electron display) and (b) third electrochemical treatment (backscattered electron display). 
electrochemical treatment. SEM images of the surfaces are shown in Figure 3 after the second and third treatments. After the third treatment bevelling of the grain boundaries became extensive (Figure 3(b)) and cracks even became apparent in the oxide within the FIB pre-treated area under higher resolution. The differences between the area pre-treated by FIB and that untreated area are apparent with much greater growth of micron-scale crystallites in the untreated area. A crack opening near a grain boundary is shown in Figure 4(a) and cracks were also found to be present among the oxide crystallites outside the FIB area. FIB sectioning was then used to create a cross-section of the oxide and underlying metal in the vicinity of the crack and this is shown in Figure 4(b). The oxide shows internal cracking as well as an extension of the crack into the grain boundary in the underlying alloy. One possibility is that expansion of the oxide because of its Pilling-Bedworth factor could have led to an initiation of a crack in the boundary. The surface oxide was depth profiled using Auger Electron Spectroscopy and was determined to be about $80 \%$ nickel oxide/20\% chromium oxide. The structure of the oxides would be expected to be $\mathrm{NiO}$ and $\mathrm{Cr}_{2} \mathrm{O}_{3}$ based on the solution $\mathrm{pH}$ and temperatures used in the autoclave [14].

(a)

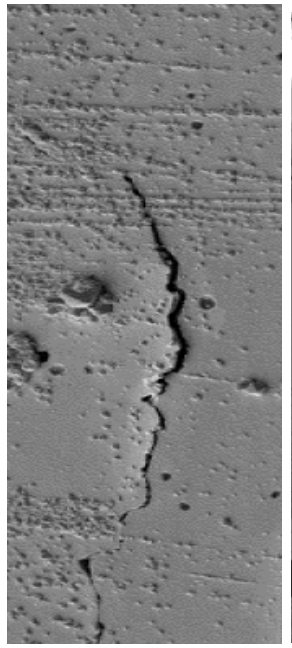

(b)

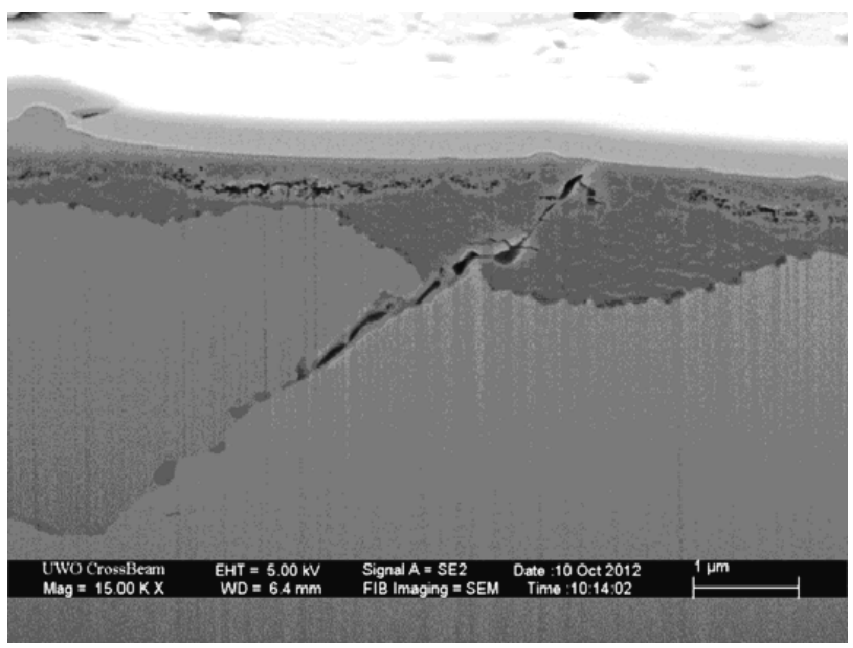

Figure 4: SEM images of a crack in the surface oxide following the third electrochemical exposure (a) plan view back scattered electron image of the crack within the FIB pre-treated area (b) secondary electron image of a FIB cross section of the same crack.

The above studies took place within the small area on the flat that had been analysed on three successive occasions by PXM (after each electrochemical treatment). The Laue diffraction maps of crystallographic orientation in the same proximate areas are shown in Figure 5. The colours shown represent crystallographic poles whose angular relationship is shown in (d). The black 

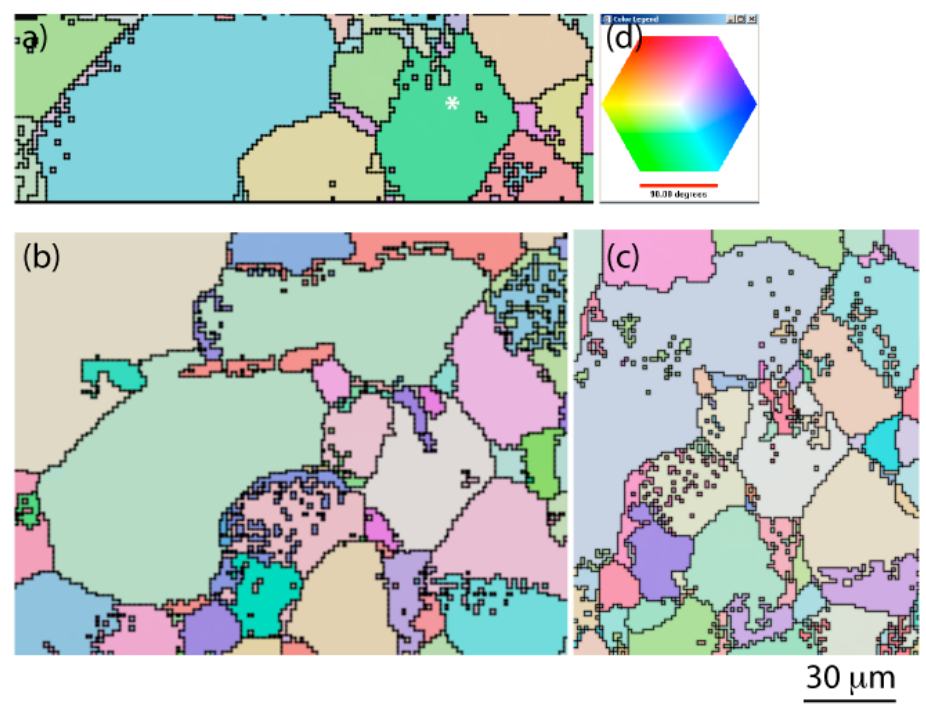

Figure 5: Crystallographic orientation maps for the same area on the polished Alloy 600 flat following: (a) $6 \mathrm{~h}$ (b) $12 \mathrm{~h}$ and (c) $24 \mathrm{~h}$ of electrochemical exposure (see text).

lines represent changes in angular orientation of $>5$ degrees. The pole figure for individual grain faces changes between exposures because the sample orientation with respect to the $\mathrm{x}$ ray beam has changed somewhat with each analysis. The length scales are only approximately the same. A correct scale bar is shown for (c). The same grain shapes can be recognised in these maps as well as in Figure 3. These maps represent information from an average of several microns into the surface. The overall shape of most of the grains did not change throughout the treatment; however, there was the notable addition of small phases along many grain boundaries. Their exact origin is not understood but it is believed that they are associated with the growth of oxide phases at or near the grain boundaries.

The local elastic strain tensors are mapped for these areas in Figures 6 and 7. Figure 6 compares changes in the composite (von Mises) elastic strains. This strain value represents the root mean square of all 6 strain tensors are determined for each pixel in the image; the volume element measured is actually $1 \mathrm{x} 1 \mathrm{x} \sim 10$ microns. The regions covered by each scan were not quite the same so the Figure shows only those equivalent regions. In Figure 6(a) the central grain marked with an * is also identified in the images in Figures 3 and 5.

After the initial 6h corrosion process most local strains are confined to the interior of grains and probably result from forging and mechanical surface preparation. In fact, the common area shown here was unusually strained compared to other regions of the sample for this treatment. After the second $6 \mathrm{~h}$ corrosion process (Fig. 6b)), there is a distinct tensile strain along the grain boundary near the centre of the map (see arrow). By contrast the areas of high strain intensity in the central grain in Figure 6a) diminished and the remaining 
regions are generally low in composite strain intensity. After the third corrosion treatment for a total of 24h, the composite strain map in Figure 6c) shows an increase in local strain intensity; particularly notable is the one of the grains forming the boundary that was strained from the previous exposure. Some other grain boundaries, both within (see arrow) and outside the field of this image show a marked increase in local composite elastic strain after the third electrochemical treatment.

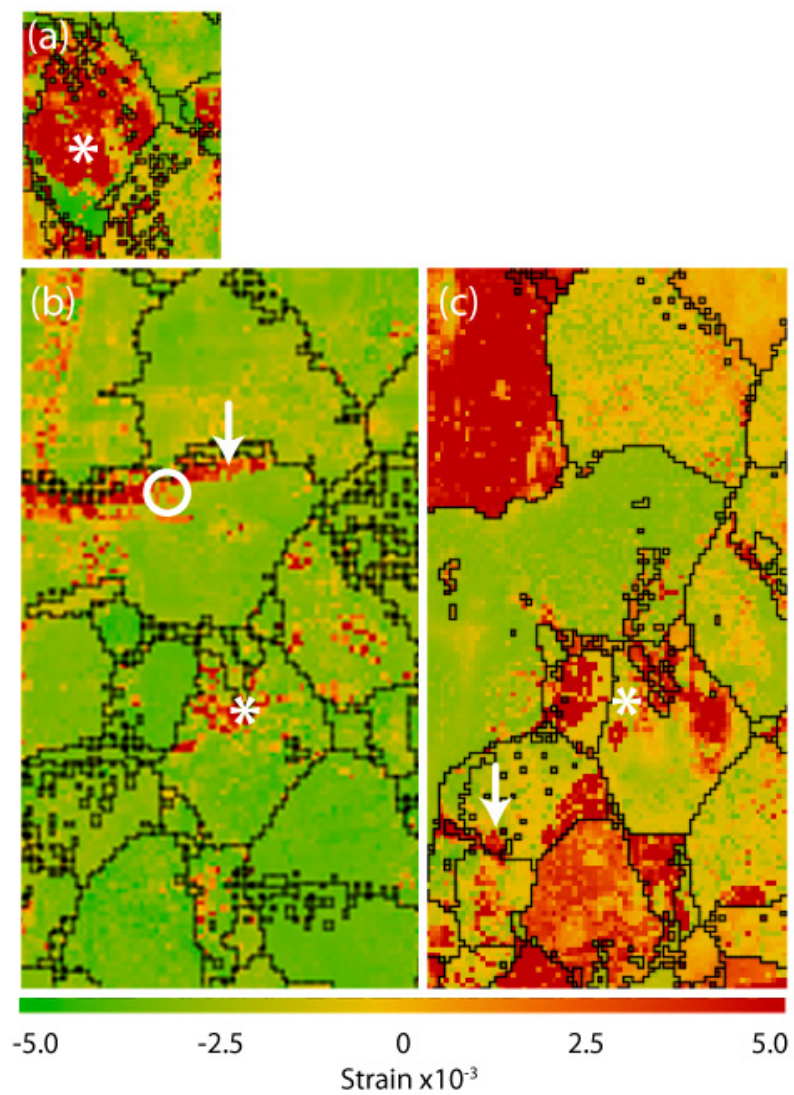

Figure 6: Composite (von Mises) elastic strain maps for the same area of the Alloy 600 flat that had been corroded for periods of (a) 6h, (b) $12 \mathrm{~h}$ and (c) $24 \mathrm{~h}$. The intensity scale is unidirectional. The dark lines indicate changes to orientation by $>5$ degrees as in the previous Figure. An * identifies the same grain in each figure as well as in Figure 3. A + indicates the region where the FIB section shown in Figure 4 was done. Arrows indicate regions of high grain boundary strain in (b) and (c). 
The elastic tensile strain along the $\mathrm{z}$ axis (into the plane of the paper) is compared in Figure 7 for approximately the same region of the flat for the second and third corrosion treatments. After the second treatment tensile strain is confined to the grain boundary region discussed above (see arrow). After the third treatment much of the entire region (and beyond) exhibits tensile strain as well as the same boundaries as before. This could possibly result from stresses caused by the growth of the oxide overlayer. Some strains in other directions within the boundary region are compressive and are probably caused by local oxide growth within the grain boundary or above it.

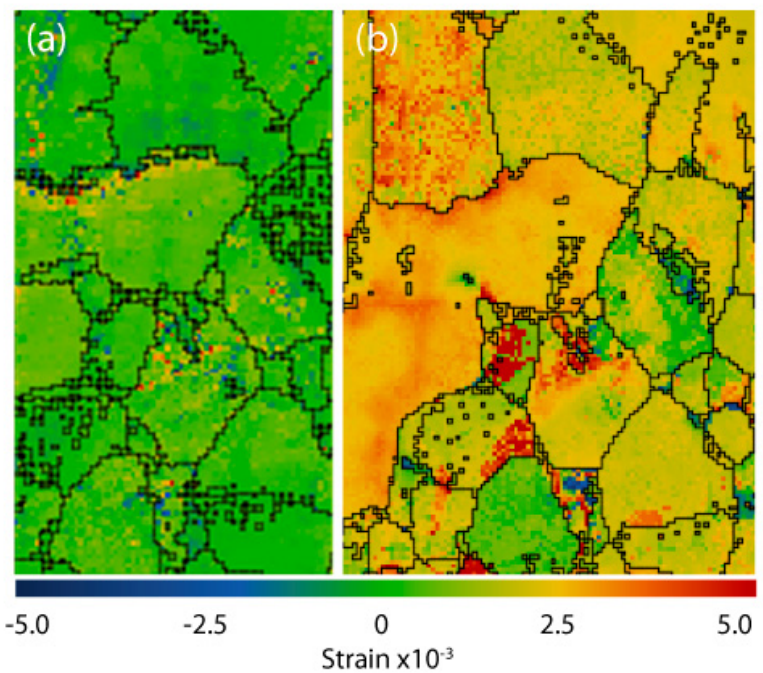

Figure 7: Comparison of strain intensity in the $\mathrm{z}$ direction after the second and third electrochemical treatments. Blue coloration indicates and compressive strain and red coloration a tensile strain.

The absolute mis-orientation maps after the second and third treatments are found in Figure 8. Of particular note is the similarity in the distributions of low levels of mis-orientations thus suggesting that these features are much less altered be the processes of corrosion than are the elastic strain distributions. As well, there is little evidence of elevated mis-orientation in the grain boundary region that exhibits high elastic strain in Figures 6 and 7. While the distribution of the mis-orientation is maximized by proximity to grain boundaries and particularly to triple points, no unusual levels of mis-orientation were found near the grain boundary that displayed high elastic stains. 


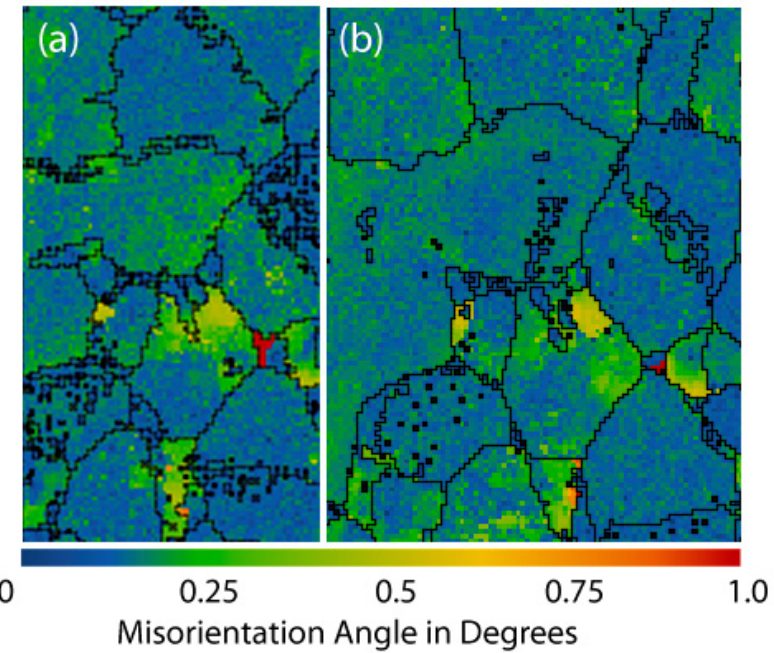

Figure 8: Comparison of absolute mis-orientation distributions for the same area of the flat Alloy 600 surface after the second and third corrosion treatments: (a) second treatment (b) third treatment.

\section{Conclusions}

This paper has been primarily concerned with the relationship between the process of stress corrosion cracking and its detection by an $\mathrm{x}$ ray diffraction technique that provides a measure of elastic strain within the nexus of the crack origins. Our previous analysis of crack propagation conditions have suggested that changes in local elastic strain were easier to associate with crack opening that were the plastic strain changes as measured by Laue-based mis-orientation Electron Beam Scattering Diffraction[15] with its shorter information depth may be more sensitive to crack opening. In this study two different forms of cracking were studied: one where the metal was pre-stressed; the other where all stresses originated from the chemical products of the corrosion process itself. In both cases it has been possible to suggest the presence of proto-cracks and actual cracks whose strain properties relate to the measured accumulation of unusual levels of stress/strain by Laue micro-diffraction.

The possible roles of surface oxide growth in the initiation of inter-granular cracking have been advanced here by the SEM observations of a compact surface oxide on the corroded alloy that undergoes some degree of stress-related cracking itself; this is believed to contribute to, if not initiate, the inter-granular cracking in the underlying alloy. 


\section{Acknowledgements}

We are grateful for the financial assistance of NSERC and the UNINE program of the CANDU Owners Group. The Advanced Light Source is supported by the Director, Office of Basic Energy Sciences of the USDOE under Contract No. DE-AC02- 05CH11231. We thank Dr J. Chao for some experimental assistance and Dr S. Ramamurthy for providing the Auger depth profile.

\section{References}

[1] Chung, J.S. and Ice, G.E., Journal of Applied Physics, 86, pp. 5249-5261, 1999.

[2] Tamura N., McDowell, A.A., Spolenak, R., Valek, B.C., Bravman, J.C., Brown, W.L., Celestre, R.S., Padmore, H.A., Batterman, B.W. and Patel, J.L., J. Synchrotron Rad., 10, p. 137, 2003.

[3] Barabash, R.I., Ice, G.E. and Walker, F.J., Journal of Appl. Phys., 93, pp. 1457-1465. 2003.

[4] Levine, L.E., Larson, B.C., Yang, W., Kassner, M.E., Tischler, J.Z., DelosReyes, M.A., Fields, R.J. and Liu, W., Nature Materials, 6, pp. 219-221 2006.

[5] Ohashi, T., Barabash, R.I., Pang, J.W.L., Ice, G.E. and Barabash, O.M., Int. J. Plasticity, 25, p. 920, 2009.

[6] Chao, J., Suominen Fuller, M.L., McIntyre, N.S., Carcea, A.G., Newman, R.C., Kunz, M. and Tamura, N., Acta Materialia, 60, pp. 781-792, 2012.

[7] Chao, J., Suominen Fuller, M.L., Sherry, N., Qin, J., McInyre, N.S., Ulaganathan, J., Carcea, A.G., Newman, R.C., Kunz, M. and Tamura, N., Acta Materialia, 60 pp. 5508-5515, 2012.

[8] Stahle, R.W. and Gorman, J.W., Corrosion, 51 pp. 931-994, 2003.

[9] Rebak, R.B. and Szklarska-Smialowska, S., Corrosion Science, 38, pp. 971-988, 1996.

[10] Kamaya, M., Mater. Charact., 60, p. 125, 2009.

[11] Hur, D.H, Kim, J.S., and Baek, JS, Corrosion, 58, p. 1031, 2002.

[12] Sherry, N., Qin, J., Suominen Fuller, ML, Xie, Y., Mola, O., Bauer, M, McIntyre, N.S , Maxwell, D., Liu, D., Matias, E. and Armstrong, C., Anal. Chem., 84, pp. 7283-7291, 2012.

[13] Chao, J., Mark, A., Fuller, M.L.S., McIntyre, N. S., Holt, R.A., Klassen, R.S., and Liu, W., Mater. Sci. Eng., A524, pp. 20-27, 2009.

[14] Payne, B. P. Keech, P. and McIntyre, N.S. manuscript in preparation.

[15] Shidmore, T., Buchheit R.G. and Juhas, M.C., Scripta Materialia, 50, pp. 873-887, 2004. 